Saclay, T94/078

Orsay, LPTHE 94/58

(June 1994)

\title{
INTERMITTENCY AND EXOTIC CHANNELS
}

\author{
A. Bialas* \\ LPTHE, Bâtiment 211, Université Paris-Sud \\ F-91405 Orsay, FRANCE \\ R. Peschanski \\ CEA, Service de Physique Théorique, CE de Saclay \\ F-91191 Gif-sur-Yvette Cedex, FRANCE
}

\begin{abstract}
It is pointed out that accurate measurements of short-range two-particle correlations in like-charge $K \pi$ and in $\pi^{0} \pi^{0}$ channels should be very helpful in determining the origin of the "intermittency" phenomenon observed recently for the like-charge pion pairs.
\end{abstract}

Submitted to Phys. Rev. D (Brief Reports)

* On leave from: Institute of Physics, Jagellonian University, Reymonta 4, PL-30 059 Cracow, POLAND 
It is now well established $[1,2]$ that the correlations between two pions at small relative momenta observed in different hadron-induced processes of multiparticle production are drastically different for the like and unlike pairs. The like pairs show the so-called "intermittency" [3], i.e. a strong (probably power-law) rise of the correlation function at small $Q^{2}$, where

$$
Q^{2}=\left(p_{1}-p_{2}\right)^{2}=\left(p_{1}+p_{2}\right)^{2}-4 m_{\pi}^{2}
$$

is the square of the difference of particle momenta. This phenomenon finds a natural explanation in terms of quantum interference between identical particles (HBT correlations [4]) which is now commonly accepted $[1,2,5]$.

This interpretation implies that the observed correlations have "geometrical" origin, i.e. they are related to the space-time structure of the volume from which the pions are emitted [6,7]. Before this picture is accepted, however, it is important to discuss if there perhaps exists other dynamical explanations for this phenomenon. In fact, it was already a long time ago [8] pointed out and recalled recently [9], that these effects can be related to another remarkable difference between $\pi^{ \pm} \pi^{ \pm}$and $\pi^{+} \pi^{-}$channels, namely their resonance structure. While the $\pi^{+} \pi^{-}$system contains many resonances, they are absent in the "exotic" $\pi^{ \pm} \pi^{ \pm}$channels.

It is of course an entirely open question if this difference implies or not a different behaviour of $\pi \pi$ correlations at very low invariant mass. However, there exist an argument suggesting that this may actually be the case [8]. It can be summarized as follows.

In the intermediate energy region, the amplitudes describing the $\pi \pi$ cross-section are known to be dominated by exchange of Regge singularities in the $t$-channel. The principle of duality implies that in the exotic channel the contribution of leading Regge poles exactly cancel each other ${ }^{1}$. Consequently, an exotic channel is dominated by exchange of singularities with low intercept $\alpha_{E}(0)$. It follows that the energy-dependent part of the $\pi^{ \pm} \pi^{ \pm}$ cross-section is expected to fall with increasing invariant mass of the $\pi \pi$ system following the power law

$$
\sigma \sim\left(M^{2}\right)^{\alpha-1}=\left(p_{1}+p_{2}\right)^{2(\alpha-1)}
$$

with $\alpha=\alpha_{E}(0) \leq 0$.

1 We do not consider the Pomeron exchange contribution, as it is irrelevant for shortrange correlations we are interested here. 
Since there are no resonances in the exotic channel, this smooth behaviour is expected to hold even at relatively low energies. It is therefore perhaps not unreasonable to speculate that it continues even down to the threshold ${ }^{2}$. This possibility is particularly suggestive, when compared to the data of Refs.[8,9] which show precisely the same power law ${ }^{3}$ at small and medium $M^{2}$. In contrast, for non-exotic $\pi^{+} \pi^{-}$channel the leading Regge poles do contribute, the intercept $\alpha=\alpha_{R}(0) \simeq 1 / 2$ is much higher and, consequently, the expected $M^{2}$ dependence much weaker than in (2). Even more important, the presence of prominent resonances in the $s$ channel disturbs significantly the simple power-law behaviour and thus precludes its trivial continuation to the threshold.

This completes the argument. Admittedly, it is not very convincing as it requires a rather bold extrapolation. We feel, however, that it should not be entirely dismissed, particularly in view of the experimental evidence shown in [8] and [9]. Indeed, if one can (at least partly) explain the difference between like-change and unlike-change $\pi \pi$ correlations without invoking quantum (HBT) interference, the interpretation of the existing data may be profoundly modified. Therefore it seems important to check carrefully this possibility.

The main point of the present note is to indicate that there exists a feasible way to test these ideas and perhaps even to give access to a relative strength of the effects of quantum (HBT) interference and those of the exotic nature of the like-charge $\pi \pi$ interactions. The idea is there exist particle combinations which are either

(a) exotic but not identical

(b) identical but non-exotic

In the category (a) one can list $K^{+} \pi^{+}$and $K^{-} \pi^{-}$pairs. Since the particles are not identical, quantum interference cannot be responsible for short-range correlations. Therefore if the effect is present in $K^{ \pm} \pi^{ \pm}$channels it is probably due to their "exoticity".

In the category (b) one has $\pi^{0} \pi^{0}$ channel. It is formed by identical particles, so if quantum interference is at work we expect a pattern similar to $\pi^{+} \pi^{+}$channel. On the

2 Of course the phase-space factors at the threshold must be corrected for. This, however, is to a large extent taken care of, when one considers normalized correlation functions [8].

3 A closer look at the data $[1,2]$ shows that they follow a power law in $Q^{2}$ rather than $M^{2}$. This is not a serious difficulty: the asymptotic formula (2), justified at large $M^{2}$ cannot distinguish these two possibilities. 
other hand $\pi^{0} \pi^{0}$ system forms $I=2$ with probability $2 / 3$ and $I=0$ with probability $1 / 3$. Only $I=2$ state is exotic and thus if the resonance structure is responsible we expect a weaker short-range correlation effect than in $\pi^{+} \pi^{+}$system.

The early data of NA22 coll.[10] and the more recent data of Aleph coll. [11] indicate that the very short range correlations in the $K \pi$ exotic channels, if present at all, are substantially weaker than in the $\pi \pi$ channel. However, we feel that a precise comparison of the behavior at very small and at medium $Q^{2}$ is necessary to assess correctly the role of exoticity in the $K \pi$ channel.

To summarize, we suggest that accurate measurements of short-range correlations in $K \pi$ and $\pi^{0} \pi^{0}$ channels, should help to determine the origin of the phenomenon of "intermittency". It seems to us important to clarify this point before one can safely conclude that the present data can be attributed solely to the effect of quantum (HBT) interference.

\section{ACKNOWLEDGEMENTS}

We would like to thank Wolfram Kittel for the correspondence about data and Andrzej Krzywicki for interesting comments. This research was supported in part by the KBN grant 200929101. 


\section{REFERENCES}

1 F. Mandl and B. Buschbeck, Vienna-preprint HEPHY-PUB-590-93 (May 1993); and Proc. XXII Int. Symp. on Multiparticle Dynamics, Santiago de Compostela Spain, 1992, p.561. A. Pajares (ed.) World Scientific, Singapore.

2 N.A. Agababyan et al., NA 22 coll., Z. Phys. C59 (1993) 405.

3 A. Bialas and R. Peschanski, Nucl. Phys. B273 (1986) 703.

For a recent review, see E.A. DeWolf, I.M. Dremin and W. Kittel, to be published in Phys. Rep. C.

4 R. Hanbury-Brown and R. Q. Twiss : Phil Mag. 45 (1954) 663; For a review see D.H. Boal et al., Rev. Mod. Phys. 62 (1990) 553.

5 H. Bächler et al., NA 35 Coll., Zeit. Phys. C61 (1994) 551.

6 P. Grassberger, Nucl. Phys. B120 (1977) 231.

7 A. Bialas, Acta Phys. Pol. B23 (1992) 561.

8 E.L. Berger, R. Singer, G.M. Thomas and T. Kafka, Phys. Rev. D15 (1977) 206.

9 I.V. Ajinenko et al., NA22 Coll., Zeit. Phys. C61 (1994) 567.

10 M. Adamus et al., NA22 Coll., Zeit. Phys. C37 (1988) 347, fig.3 e,f.

11 D. Decamp et al., NA22 Coll., Zeit. Phys. C54 (1992) 75, fig.7. 